\title{
Arrival directions of the highest-energy cosmic rays detected by the Pierre Auger Observatory
}

\author{
Ugo Giaccari ${ }^{* a}$ for the Pierre Auger Collaboration ${ }^{b}$ \\ ${ }^{a}$ Instituto de Física, Universidade Federal do Rio de Janeiro, Brazil \\ now at Centro Brasileiro de Pesquisas Físicas, Rio de Janeiro, Brazil \\ ${ }^{b}$ Observatorio Pierre Auger, Av. San Martín Norte 304, 5613 Malargüe, Argentina \\ E-mail: auger_spokespersons@fnal.gov \\ Full author list: http://www.auger.org/archive/authors_icrc_2017.html
}

\begin{abstract}
We present the analysis of the arrival direction distribution of the highest-energy cosmic rays detected at the Pierre Auger Observatory. The data collected to date, with a total exposure of approximately $90,000 \mathrm{~km}^{2} \mathrm{sr}$ yr, allow us a precise characterisation of the patterns in the arrival direction distribution.

An update of the two largest departures from isotropy already reported is discussed here. The region of the sky close to the direction of Centaurus A is analyzed and the correlation with the positions of the most luminous AGNs detected by Swift-BAT is investigated.

We also examine the correlation of our highest energy events with two populations of extragalactic sources of gamma rays, namely star-forming galaxies and AGNs motivated by the observations of the Fermi-LAT satellite. In this new search, a likelihood analysis is adopted which gives weights to the individual sources in proportion to their fluxes. The same flux-weighted search for an angular correlation with the extragalactic sources listed in the Swift-BAT catalog is also performed.

The significances of the excesses around Centaurs A and the most luminous AGNs detected by Swift-BAT have increased and reached to the $3 \sigma$ level approximately. An excess at $2.7 \sigma$ level is found for the gamma-ray AGNs while for the star-forming galaxies there is a $4 \sigma$ deviation from isotropy for energies greater than $39 \mathrm{EeV}$ at an intermediate angular scale of $13^{\circ}$.
\end{abstract}

35th International Cosmic Ray Conference - ICRC2017

10 - 20 July, 2017

Bexco, Busan, Korea

*Speaker. 


\section{Introduction}

The identification of the astrophysical objects able to accelerate ultra high-energy cosmic rays (UHECRs) is a longstanding question in astrophysics. Patterns in the arrival direction distribution of UHECRs over the celestial sphere could provide significant clues for clarifying the origin of these particles.

Cosmic rays with energies above the steepening of the energy spectrum at $\sim 4 \times 10^{19} \mathrm{eV} \mathrm{[1]}$, give us information about UHECR sources within the local Universe up to $\sim 200-300$ Mpc. Particles in this energy range reach us from very few hundred Mpc due to the energy losses in the interactions with the cosmic microwave and infrared backgrounds. With the isotropic distant sources removed at energies above the aforementioned steepening in the spectrum, it is feasible to detect anisotropies in the arrival direction distribution reflecting the inhomogeneities in the distribution of the local sources.

This paper deals with the study of the arrival directions of the highest-energy cosmic rays detected at the Pierre Auger Observatory. Despite the tiny flux of particles in this energy range, the huge collecting area of the Observatory together with its ability to detect air showers up to $80^{\circ}$ in zenith offer the possibility to study the arrival direction distribution of the UHECRs with unprecedented statistics.

At the Pierre Auger Observatory, data collected through $31^{\text {st }}$ March 2014 have been subjected to different searches for anisotropies for different energy thresholds between 40 and $80 \mathrm{EeV}$ and within different angular windows, between $1^{\circ}$ and $30^{\circ}$ [2]. Searches for intrinsic anisotropies have been performed, as well as cross-correlations with the directions of candidate UHECR accelerators. Out of all the performed searches the two largest deviations from isotropy, both with posttrial probability $\sim 1.4 \times 10^{-2}$, have been found for an energy threshold of $58 \mathrm{EeV}$ when looking into the region of the sky within $15^{\circ}$ from the location of Centaurus A (Cen A) and when considering the cross-correlation with the most luminous active galactic nuclei (AGNs) detected in X-ray from the Swift-BAT mission [3].

In this report we first update these two searches with respect to that already reported using the conventional correlation methods [2]. We also discuss the correlation of our highest energy events with two populations of extragalactic sources of gamma rays: AGNs and star-forming galaxies motivated by the observations of the Fermi-LAT satellite.

With these two classes of astrophysical objects we search not only for angular correlations with the directions of the objects on the sky but also with their flux through appropriate weights. The same analysis technique, for an angular and flux correlation, is also discussed for the extragalactic objects listed in the Swift-BAT catalog.

All the searches presented here are a posteriori explorations. Numerous studies have been performed in the past with Auger data within and outside the collaboration. This makes difficult to evaluate a proper penalty factor for all the previous searches.

\section{The Observatory and the datasets}

The Pierre Auger Observatory [4] is the world's largest cosmic ray observatory at energies above $10^{17} \mathrm{eV}$. It is located in the Province of Mendoza, Argentina. The site is placed at an average latitude of $\sim 35.2^{\circ} \mathrm{S}$ and a mean altitude of $\sim 1400 \mathrm{~m}$ above the sea level. The Observatory is a 
hybrid detector combining the information from a large surface detector array (SD) and a fluorescence detector (FD). The SD, spread over an area of $3000 \mathrm{~km}^{2}$, is composed of a baseline array of 1600 water-Cherenkov detectors separated by $1500 \mathrm{~m}$ in a triangular grid, and a smaller nested array of 49 additional detectors spaced by $750 \mathrm{~m}$ covering an area of $24 \mathrm{~km}^{2}$. The FD consists of 27 telescopes at five peripheral buildings viewing the atmosphere over the array. The combination of the two detection techniques provides a unique handle for precision energy calibration and the understanding of reconstruction uncertainties of energy of the cosmic rays. The FD operates only on clear, moonless nights, so its duty cycle is about $15 \%$. On the other hand, the SD has a duty cycle close to $100 \%$. The unique characteristics of the SD, the huge collecting area, the sensitivity to detect showers up to $80^{\circ}$ and the long data collection period, provide the accumulation of a large body of data for anisotropy studies.

In this analysis we consider showers observed with the SD between the $1^{\text {st }}$ January 2004 and the $30^{\text {th }}$ April 2017 with zenith angle $\theta \leq 80^{\circ}$ and reconstructed energy $E \geq 40 \mathrm{EeV}$. The event reconstruction procedure depends on the zenith angle of the air shower. Events with zenith angle $\theta \leq 60^{\circ}$ are labeled as vertical, while those with $60^{\circ}<\theta<80^{\circ}$ are labeled as inclined ones. A detailed description of the data selection criteria can be found in [2]. The angular uncertainty is better than $1^{\circ}$ above $10 \mathrm{EeV} \mathrm{[5]} \mathrm{and} \mathrm{the} \mathrm{final} \mathrm{energy} \mathrm{estimation} \mathrm{uses} \mathrm{the} \mathrm{cross-calibration} \mathrm{with} \mathrm{the}$ FD providing a quasi-calorimetric measurement. The statistical uncertainty in the energy determination is better than $12 \%$ [6] above $10 \mathrm{EeV}$ and the systematic uncertainty in the absolute energy scale is $14 \%$ [7]. In this energy range the SD is fully efficient, the total exposure amounts to 71,070 and $18,650 \mathrm{~km}^{2} \mathrm{sr}$ yr for the vertical and inclined samples respectively. The directional exposure relies on geometrical considerations and can be expressed analytically. Unfolding factor corrections as derived in the framework of the energy spectrum studies [8] are included in the exposures of vertical and inclined events, to account for the different migration effects due to the different energy resolutions of each data stream.

\section{Update of the correlation studies with Centaurus A and with the most luminous AGNs of the Swift-BAT catalog}

Among all the searches performed so far [2] the two most important deviations from isotropy have been found looking in the direction of Cen A and when considering the most luminous AGNs of the Swift-BAT catalog. In this section we update these two analyses. The search for excess around Cen $\mathrm{A}$ is performed counting the number of observed $\left(n_{\mathrm{obs}}\right)$ and expected ( $\left.n_{\text {exp }}\right)$ events from an isotropic flux within circles of radius $\psi$ centered around this AGN position above a given energy threshold $\mathrm{E}_{\mathrm{th}}$. Then we computed the cumulative binomial probability $P$ to measure $n_{\mathrm{obs}}$ or more events given $n_{\text {exp }}$. For quantifying the cross-correlation between our highest energy events and the most luminous AGNs of the Swift-BAT catalog we use the standard two-point correlation function. We count the number of pairs between the event directions and the positions of the astrophysical objects having an angular separation less than a given angle $\psi$. We search for excesses of pairs above the isotropic expectation by calculating the fractions of isotropic simulations, $f$, having an equal or higher number of pairs than the data.

In both analyses we search for the most relevant excess in different energy thresholds $E_{\text {th }}$, ranging from 40 up to $80 \mathrm{EeV}$ in steps of $1 \mathrm{EeV}$, and in the angular scale $\psi$ between $1^{\circ}$ and $30^{\circ}$ in 

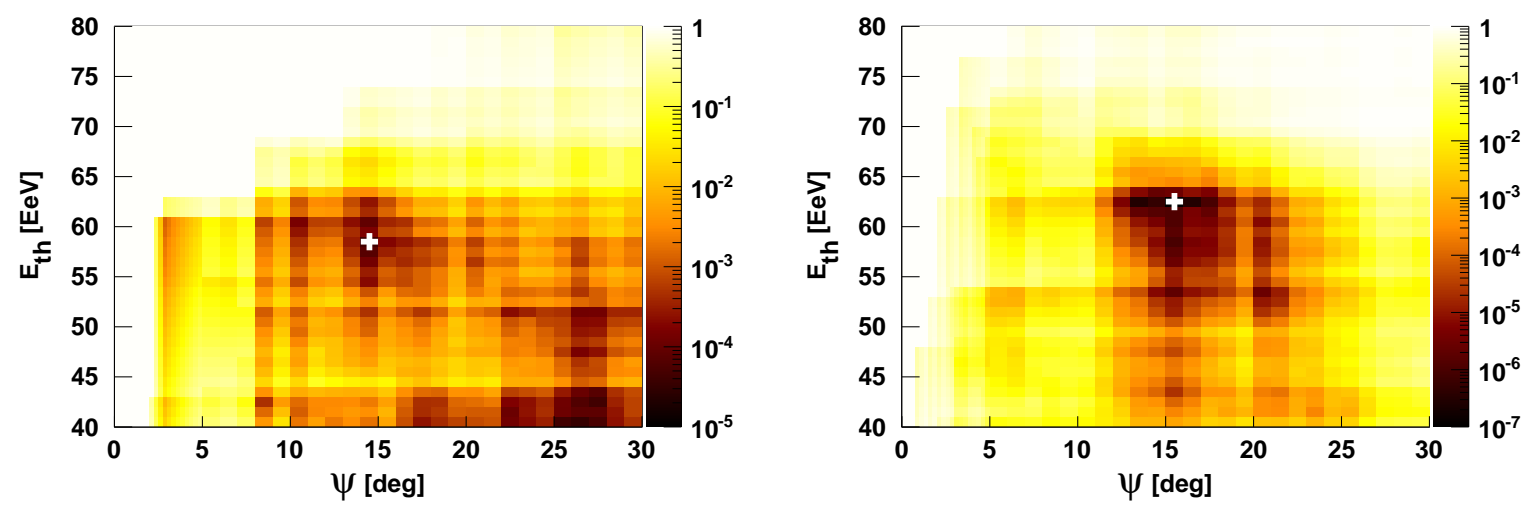

Figure 1: Left: Correlation of events with Cen $\mathrm{A}$ as a function of the angular distance $\psi$ and the energy threshold $\mathrm{E}_{\mathrm{th}}$. Right: Scan in $\left(\mathrm{E}_{\mathrm{th}}, \psi\right)$ for the cross-correlation of events with the most luminous AGNs of the Swift-BAT catalog within $130 \mathrm{Mpc}$ and brighter than $10^{44} \mathrm{erg} / \mathrm{s}$.

steps of $0.25^{\circ}$ up to $5^{\circ}$ and of $1^{\circ}$ for larger angles. The total data set for these two analyses is the combination of 650 vertical events and 170 inclined ones above $40 \mathrm{EeV}$.

For the correlations with the AGNs of the Swift-BAT catalog we select only the brightest sources by applying a cut in intrinsic luminosity. This is motivated by the expectation that the maximum energy achievable by the cosmic rays may be related to the intrinsic electromagnetic bolometric luminosity of the objects. In this case, we test the assumption that only sources intrinsically brighter than some given luminosity can accelerate cosmic rays above an energy threshold $\mathrm{E}_{\mathrm{th}}$. The scan in luminosity ranges from $L=10^{42} \mathrm{erg} / \mathrm{s}$ up to $10^{44} \mathrm{erg} / \mathrm{s}$ considering three logarithmic steps per decade. We also impose a cut in the maximum AGN distance $D$, that can vary from $10 \mathrm{Mpc}$ up to $190 \mathrm{Mpc}$ in steps of $20 \mathrm{Mpc}$.

The left panel of Figure 1 shows the correlation with Cen A. The white cross indicates the minimum value of the cumulative binomial probability, $P=1.1 \times 10^{-5}$ located at $\mathrm{E}_{\mathrm{th}}=58 \mathrm{EeV}$ and $\psi=15^{\circ}$. For these parameters there are 19 observed events in the data (out a total of 203) while $\sim 6.0$ are expected on average from an isotropic flux. The significance of this excess can be obtained by penalising for the same scan in energy and angular scale. Performing such procedure we obtain a statistical significance of $\sim 3.1 \sigma$.

The most relevant excess, corresponding to the minimum value of $f$, for the AGNs of the SwiftBAT catalog is obtained for $D=130 \mathrm{Mpc}$ and $L=10^{44} \mathrm{erg} / \mathrm{s}$. For these two parameters the right panel of Figure 1 shows the results of the scan in angle and energy threshold. The minimum value $f_{\text {min }}=1 \times 10^{-7}$ corresponds to $\mathrm{E}_{\text {th }}=62 \mathrm{EeV}$ and $\psi=16^{\circ}$, where 57 pairs are observed in data and 26.4 are expected in average from isotropy. The significance after considering the same scan anywhere in the parameter space $\left(D, L, \mathrm{E}_{\mathrm{th}}, \psi\right)$ is $\sim 3.2 \sigma$.

\section{Cross-correlation studies in relation to other catalogs}

In this section we examine the correlation of our highest energy events with two classes of astrophysical UHECR accelerators. Analyses such as that in [9] show that gamma ray emitters could meet the requirements to accelerate UHECRs. Among the nearby astrophysical objects that satisfy these conditions there are AGNs as well as star-forming galaxies even though only a handful 


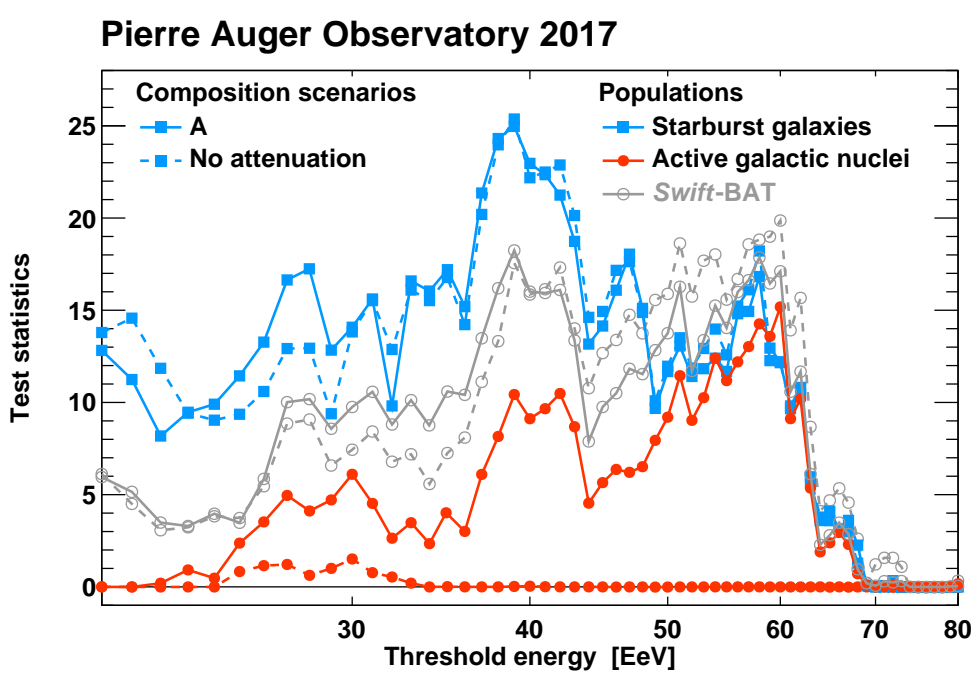

Figure 2: Test statistic as a function of the threshold energy for starburst galaxies (blue lines), gamma-ray AGNs (red lines) and Swift-BAT AGNs (gray lines). The continuous lines indicate the $T S$ values obtained accounting for attenuation as in scenario A (see text) while the dotted lines refer to the values without any attenuation.

of them have been detected in the gamma-ray band. We select active and star-forming galaxies motivated by the observations made by Fermi-LAT. Active galaxies are extracted from the 2FHL Catalog [10], which includes 360 gamma-ray sources detected above $50 \mathrm{GeV}$. Selecting only radioloud AGNs within a $250 \mathrm{Mpc}$ radius results in a list of 17 bright nearby candidates, whose integral gamma-ray fluxes between $50 \mathrm{GeV}$ and $2 \mathrm{TeV}$ are used as a proxy for the UHECR flux. For star-forming objects, we supplement the informations from Fermi-LAT with observations in the radio band, where more in-depth surveys of this population of sources have been carried out. Only a handful of star-forming galaxies have been detected in the gamma-ray band, but their gammaray luminosity has been shown to nearly linearly scale with their continuum radio flux [11]. We use their continuum emission at $1.4 \mathrm{GHz}$ as a proxy for the UHECR flux [12]. Among the 63 objects within $250 \mathrm{Mpc}$ that have been searched for gamma-ray emission [11], we select only the 23 brightest nearby objects with a radio flux larger than $0.3 \mathrm{Jy}$, noting though a posteriori that this criterion does not affect the results presented in Section 4.1. The selection procedure results in two nearly equal-size flux-limited samples: the active galaxies and the star-forming or starburst galaxies.

\subsection{Maximum-likelihood technique}

In Section 3 we investigated the correlation with the brightest AGNs of the Swift-BAT catalog under the assumption that all the selected sources contribute equally to the UHECR flux. Motivated by the expectation that brighter objects contribute more to the flux, we perform here correlation analyses by weighting the contribution of each astrophysical object to their relative flux.

Given a model of candidate sources, a probability map of the arrival distribution of cosmic rays can be obtained in the form of a smoothed density map and compared to the observed distribution of events using an appropriate test statistic $(T S)$. The smoothed maps are constructed by weight- 

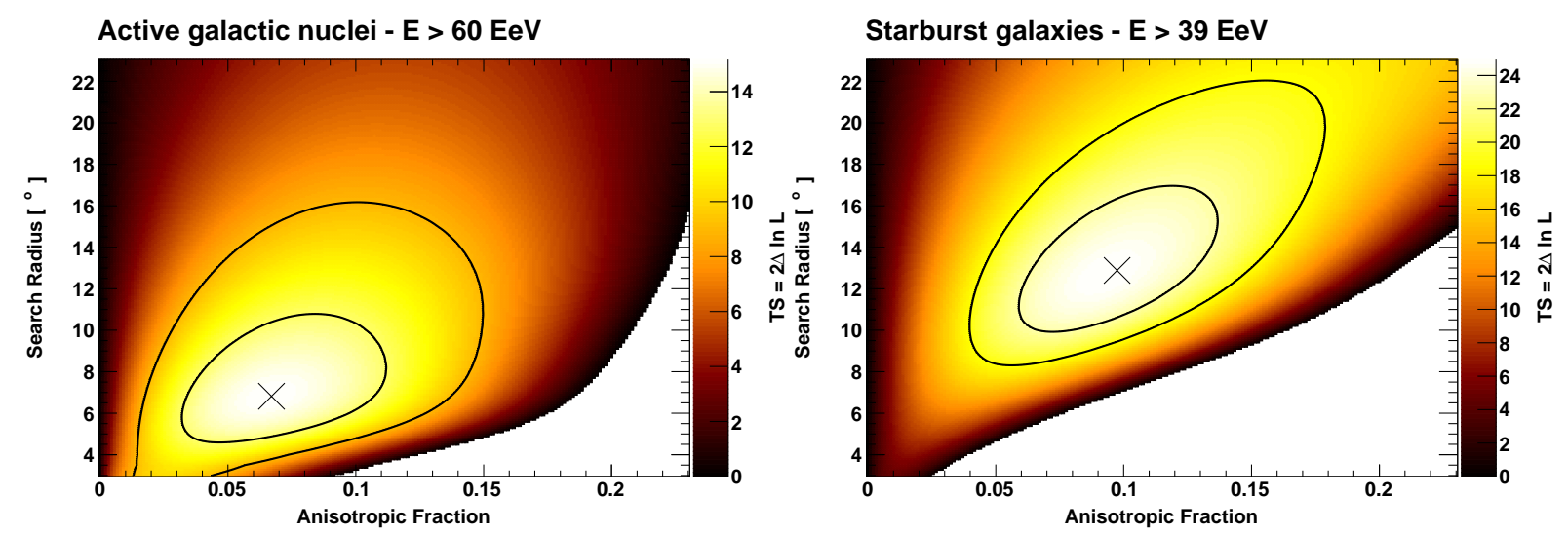

Figure 3: Test statistic as a function of the smoothing angle and anisotropic fraction for the best fit energy for the AGNs (left panel) and for the starburst galaxies (right panel). The solid lines indicate the 1, $2 \sigma$ confidence contours.

ing the objects by their relative flux, we use the gamma-ray and the radio flux for the AGNs and the starburst galaxies respectively. The contribution of each individual object is modeled with a Fisher-Von Mises distribution characterized by an angular width. This smearing angle, acting as an effective search radius, takes into account the unknown deflections of the UHECRs in the magnetic fields and constitutes the first free parameter in the analysis.

The contribution of each object is weighted also for its distance to take into account the propagation effects in the intergalactic medium. The attenuation factor used in this analysis corresponds to the mass composition scenario at sources that best reproduce the energy spectrum and the average nuclear composition measured by the Pierre Auger Observatory [13]. An isotropically distributed fraction of events is also added to the probability maps for modelling the diffuse component in the UHECR flux. This component could account for the events highly deflected by the magnetic fields (due to high electric charge and/or strong fields). The anisotropic fraction, the counterpart of the isotropic fraction, is the second free parameter in the analysis. A value of the anisotropic fraction significantly different from zero indicates that the contribution from astrophysical sources deviates strongly from a purely diffuse flux. Such maps are multiplied by the exposure, and then normalised to the total number of UHECR events.

The model predictions are compared to the data using the maximum likelihood ratio method. The likelihood function $(\mathscr{L})$ as defined in [14] is given by the product over the events of the probability map. The likelihood of the null hypothesis $\mathscr{L}_{0}$ (isotropy) is the likelihood obtained as the product over the events of the directional exposure function in the arrival directions. The likelihood ratio constitutes the test statistic (TS) used in this search analysis, $T S=2 \ln \mathscr{L} / \mathscr{L}_{0}$. According to the Wilks's theorem [15] the realizations from isotropic skies should lead to a TS that follows a $\chi^{2}$ distribution with two degrees of freedom, an expectation found to be in good agreement with simulations of isotropy.

We maximize the test statistic $T S$ as a function of two free parameters for each source population in different energy threshold $\mathrm{E}_{\text {th }}$ in steps of $1 \mathrm{EeV}$ from 20 up to $80 \mathrm{EeV}$. In this case we scan in energy down to $20 \mathrm{EeV}$ because the largest departure from isotropy has been found close to $40 \mathrm{EeV}$ for one population of gamma-ray emitters. Above $20 \mathrm{EeV}$ the total data set is the combination of 

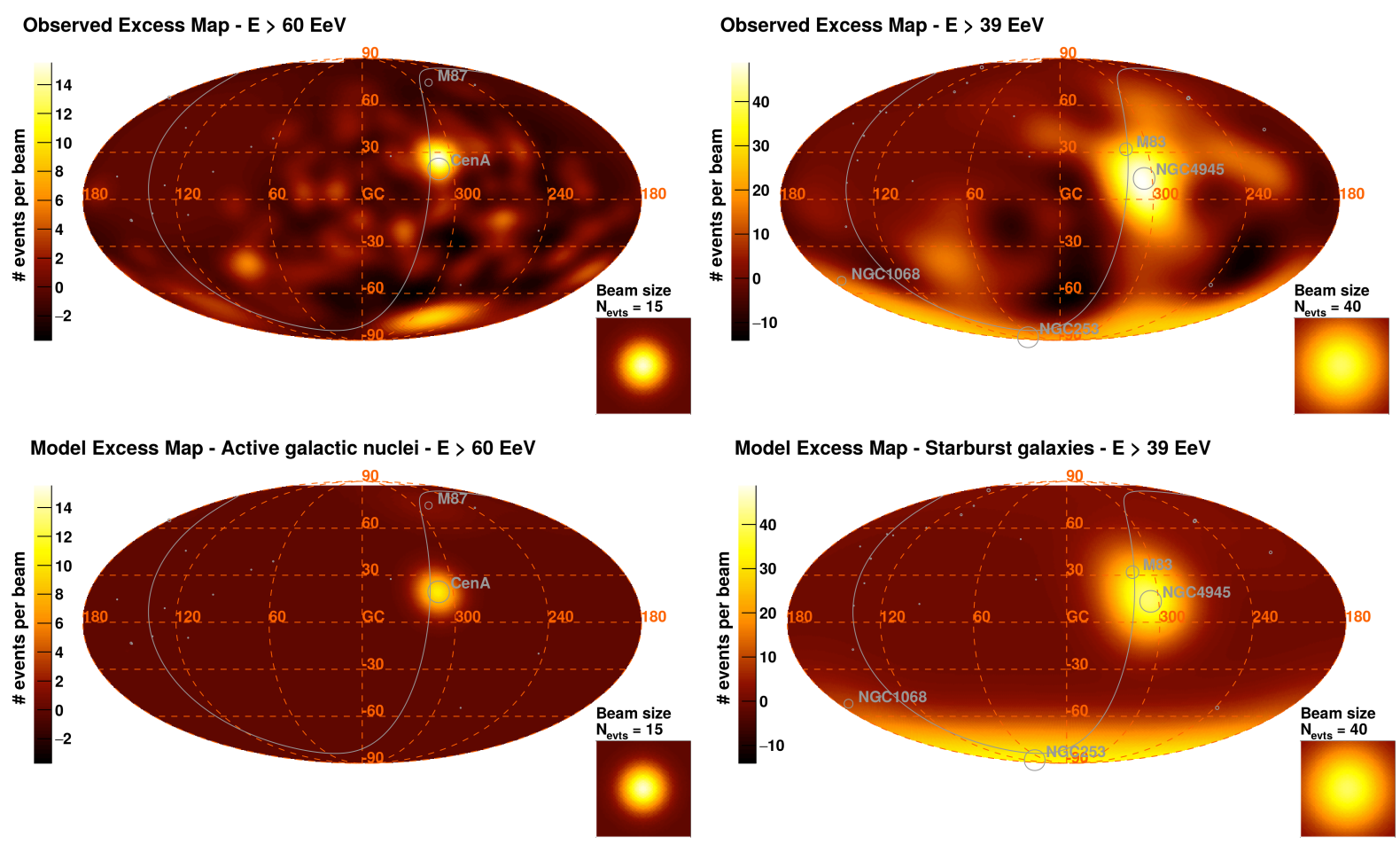

Figure 4: Observed (top) and model (bottom) excess maps obtained with the best-fit parameters for the gamma-ray AGNs (left) and for the starburst galaxies (right) in galactic coordinates.

4,396 vertical events and 1,118 inclined ones.

In Figure 2 there is the maximum value of the TS in each energy threshold for the two selected populations of gamma-ray sources. The maximum value of the test statistic for the gamma-ray AGNs, $T S=15.2$, is obtained for $\mathrm{E}_{\mathrm{th}}=60 \mathrm{EeV}$, while for the star-forming galaxies the maximum value $T S=24.9$ is reached at $\mathrm{E}_{\text {th }}=39 \mathrm{EeV}$. The same search is performed without considering any attenuation model due to the UHECR propagation. The energy losses due to the propagation have a negligible effect for the starburst galaxies, because the brightest objects are located $3 \mathrm{Mpc}$ to $20 \mathrm{Mpc}$ away. The gamma-ray AGNs are more distant objects and the impact of the selected attenuation factor is more important.

In Figure 2 we also show the same weighted search for the AGNs of the Swift-BAT catalog within $250 \mathrm{Mpc}$ with flux greater than $13.4 \times 10^{-12} \mathrm{erg} / \mathrm{s} / \mathrm{cm}^{2}$ in the X-ray band from 14 to $195 \mathrm{keV}$. In this case the maximum value of the test statistic $T S=19.9$ is found for $\mathrm{E}_{\mathrm{th}}=60 \mathrm{EeV}$ and no attenuation.

The behaviour of test statistic, for the energy threshold that maximize the likelihood ratio, is shown in Figure 3 as a function of the two fit parameters. The smearing angle and the anisotropic fraction corresponding to the best-fit parameters are $13^{\circ}$ and $10 \%$ for the starburst-galaxies and $7^{\circ}$ and $7 \%$ for the gamma-ray AGNs. The significance of the maximum value of the $T S$ can be obtained by simulating a large number of isotropic samples and by counting the fraction or random sets leading, under the scan in energy, to a greater value of the $T S$ than the data. We found an excess at $2.7 \sigma$ level for the gamma-ray AGNs, while for the starburst galaxies there is a deviation from isotropy 
at $4 \sigma$ level. The maximum $T S$ value for the gamma-ray AGN is located at $60 \mathrm{EeV}$, we see in the observed and model maps shown in Figure 4 that the major contribution come from events in the region of the sky close to Cen A. Events from this region contribute also to the maximum deviation from isotropy observed in the Swift-BAT AGNs found at $\sim 60 \mathrm{EeV}$ obtained in Section 3 and shown also in Figure 4. The events that contribute most to the starburst model are those arriving from directions close to Cen A and South Galactic pole, nearby the locations of the brightest starburst galaxies (NGC 4945, NGC 1068 NGC 253 and M83).

\section{Discussions and conclusion}

In this work we have examined with a posteriori explorations the patterns of the arrival direction distribution of the highest energy cosmic rays detected by the Pierre Auger Observatory. We have updated the two largest departures from isotropy above $40 \mathrm{EeV}$ previously reported around the direction towards Centaurus A and the most luminous AGNs of the Swift-BAT catalog. The significance with an enlarged dataset has increased to the $\sim 3 \sigma$ level for both searches. The most important excess around Centaurus A is found for cosmic rays with energies greater than $58 \mathrm{EeV}$ in an angular window of $15^{\circ}$. Around $16^{\circ}$ from the directions of the AGNs of the Swift-BAT catalog brighter than $10^{44} \mathrm{erg} / \mathrm{s}$ and within $130 \mathrm{Mpc}$ we find an excess for energies greater than $62 \mathrm{EeV}$.

We also examine the correlation of our highest energy events with two nearby populations of extragalactic gamma rays sources, namely star-forming galaxies and AGNs motivated by the observations of the Fermi-LAT satellite. A $2.7 \sigma$ excess has been found in the directions of the active galaxies, while with the starburst galaxies there is a $4 \sigma$ deviation from isotropy at intermediate angular scale.

\section{References}

[1] The Pierre Auger Collaboration, Phys. Rev. Lett. 101 (2008) 061101.

[2] The Pierre Auger Collaboration, Astrophys. J. 804 (2015) 15.

[3] W. H. Baumgartner et al., Astrophys. J. Supp. 207 (2013) 19.

[4] The Pierre Auger Collaboration, Nucl. Instrum. Meth. A 798 (2015) 172.

[5] C. Bonifazi, for The Pierre Auger Collaboration, Nucl. Phys. B Proc. Suppl. 190 (2009) 20.

[6] R. Pesce, for the Pierre Auger Collaboration, Proc. 32nd ICRC (2011), [arXiv:1107.4809].

[7] V. Verzi, for the Pierre Auger Collaboration, Proc. 33rd ICRC (2013), [arXiv:1307.5059].

[8] F. Fenu, for the Pierre Auger Collaboration, Proc. 35nd ICRC (2017), [CRI267].

[9] C. D. Dermer and S. Razzaque, Astrophys. J. 724 (2010) 1366.

[10] M. Ackermann et al. Astrophys. J. 222 (2016) 5.

[11] M. Ackermann et al., Astrophys. J. 755 (2012) 164.

[12] Y. Gao and P. M. Solomon, Astrophys. J. Suppl. Ser. 152 (2004) 63.

[13] The Pierre Auger Collaboration, JCAP 04 (2017) 038.

[14] The Pierre Auger Collaboration, Astropart. Phys. 34 (2010) 34.

[15] S. S. Wilks, Ann. Math. Statist. 9 (1938) 60. 